\title{
Measurements of atmospheric water vapor above Mauna Kea using an infrared radiometer
}

\author{
David A. Naylor*, Ian M. Chapman, Brad G. Gom \\ Department of Physics, University of Lethbridge, Alberta, Canada
}

\begin{abstract}
Astronomical arrays operating at (sub)millimeter wavelengths are seriously compromised by rapid variations in atmospheric water vapor that distort the phase coherence of incoming celestial signals. The signal received by each antenna of the array suffers a phase delay that varies rapidly with time and from antenna to antenna. Unless corrected, these distortions limit the coherence time of the array and seriously compromise its sensitivity and image quality. Building on the success of a prototype infrared radiometer for millimeter astronomy (IRMA), which operates in the $20 \mu \mathrm{m}$ region to measure the column abundance of atmospheric water vapor, this paper presents results obtained with a second generation IRMA operating at the James Clerk Maxwell telescope (JCMT) between January and July 2001. The results are compared with other measures of water vapor available on the summit of Mauna Kea, including: the JCMT SCUBA bolometer camera, the California Institute of Technology (CSO) opacity monitors, the JCMT $183 \mathrm{GHz}$ water vapor radiometer and Hilo-launched radiosonde data.
\end{abstract}

Keywords: Infrared, radiometer, water vapor, Mauna Kea

\section{INTRODUCTION}

The development of large baseline, (sub)millimeter wavelength interferometers, such as the Atacama Large Millimeter Array (ALMA) to be located high $(\sim 5000 \mathrm{~m})$ in the Chilean Andes, will provide imaging capabilities in the 10 milliarcsecond range when operating at their highest frequencies. The principle of interferometry requires that the time delays between reception of the electromagnetic wavefront at different antennae composing the array be measured accurately. Any local, line-of-sight variation in the optical path due atmospheric inhomogeneities will distort the wavefront and, if left uncorrected, lead to a misinterpretation of astronomical source structure. The factor which now limits the attainable spatial resolution of large (sub)millimeter arrays is the variation in the line-of-sight water vapor abundance which causes variations in the electromagnetic path length, and hence interferometric phase.

The principal method used to determine the line-of-sight abundance of atmospheric water vapor is the multi-channel radiometric observation of the $183 \mathrm{GHz}$ water vapor emission line ${ }^{1}$. In this method antenna brightness temperature measurements at three frequencies close to the water vapor line transition at $183.31 \mathrm{GHz}$ are fitted to a simple atmospheric emission model, whose inputs are ground level meteorological information, to derive the column abundance of water vapor, expressed in millimeters of precipitable water vapor (pwv). The principal disadvantages of this approach are the relatively low signal levels (due to the small spectral bandwidths of each radiometric channel $(\sim 1 \mathrm{GHz})$ and the inherently low radiant emission of the atmosphere in this spectral region) and the risk of radio frequency interference from the $183 \mathrm{GHz}$ local oscillator located within the receiver cabin of the antenna.

\section{IRMA CONCEPT}

Measurements of the atmospheric transmission above Mauna Kea in the $20 \mu \mathrm{m}$ atmospheric window, using a high resolution infrared Fourier transform spectrometer, have shown that over a large part of this region the atmospheric absorption, and hence emission, is dominated by pure rotational transitions of water vapor ${ }^{2}$. Detailed modeling shows that it is possible to select a continuous region containing several hundred water vapor lines, the vast majority of which are unsaturated for column abundances of $\leq 1 \mathrm{~mm}$ pwv above Mauna Kea, Hawaii. At lower altitude sites the lines in this spectral region become broader and saturated and thus the technique becomes less sensitive or unusable.

An infrared approach to water vapor measurement is attractive for several reasons: Firstly, since the wavelength of 20 $\mu \mathrm{m}$ lies near to the peak of the Planck curve for typical atmospheric temperatures, the spectral radiance from atmospheric water vapor at infrared wavelengths is $\sim 3$ orders of magnitude greater than at radio frequencies. Secondly,

\footnotetext{
*Naylor@uleth.ca; phone 1403 329-2741; fax 1403 329-2057; Dept. of Physics, 4401 University Drive, Lethbridge, AB, Canada, T1K 3M4
} 
the infrared radiometer uses a spectral bandwidth $\sim 3$ orders of magnitude greater than the radio frequency technique. The resulting increase in flux can be traded in terms of more sensitive measurements, faster operation, smaller instrument size, or some combination thereof. Thirdly, infrared photoconductive detectors offer high operating speeds, stability, and simple electronics. Finally, being a passive device, an infrared radiometer can be placed in close proximity to sensitive radio frequency instrumentation without risk of interference.

A prototype infrared radiometer for the measurement of atmospheric water vapor (IRMA I) was developed and tested at the James Clerk Maxwell Telescope (JCMT) in December 1999. The key features of the instrument are shown in figure 1. The radiometer consisted of a liquid nitrogen cooled Mercury-Cadmium-Telluride (MCT) photoconductive detector that alternately viewed the atmosphere and ambient and liquid nitrogen blackbody references by means of a stationary parabolic mirror and a scanning plane mirror. The scanning mirror provided a range of observable zenith angles from 0 to 70.38 degrees in steps of 0.18 degrees, corre sponding to an airmass range from 1 to 3 . The optical input to the detector was chopped at $200 \mathrm{~Hz}$ by a reflective chopper blade so that the detector was alternately presented views of the atmosphere (or the blackbodies) and a reflected view of its own cold environment. The modulated detector signal was amplified, synchronously detected by means of a lock-in amplifier, digitized by a 12-bit analog-to-digital converter (ADC) and the resulting data was logged to a file.

The radiometer was operated in continuous scan mode in which the atmospheric emission was measured as a function of airmass, these data being referred to as skydips (a skydip from 1 to 3 airmass taking 30s). Analysis of the IRMA I skydips ${ }^{3,4}$ showed that the infrared technique holds much promise for the challenging requirements of phase correction of the next generation of (sub)millimeter interferometers. Key elements of the prototype radiometer were subsequently improved, and include: a more sensitive Mercury-Cadmium-Telluride photoconductive detector (Kolmar Technologies; http://www.kolmartech.com), a resonant grid long pass filter (this technology is well established at submillimeter and far-infrared wavelengths but has only recently been extended to shorter wavelengths ${ }^{5}$ ), a custom lock-in amplifier, and a high-resolution (24-bit) $\Delta \Sigma$ analog-to-digital converter (CS5534, Cirrus Logic Inc.; http://www.cirrus.com), which is synchronously triggered by a notch on the reflecting chopper. Finally IRMA II was modified to allow for remote operation. This paper presents results obtained with IRMA II operating at the JCMT between January and July 2001.

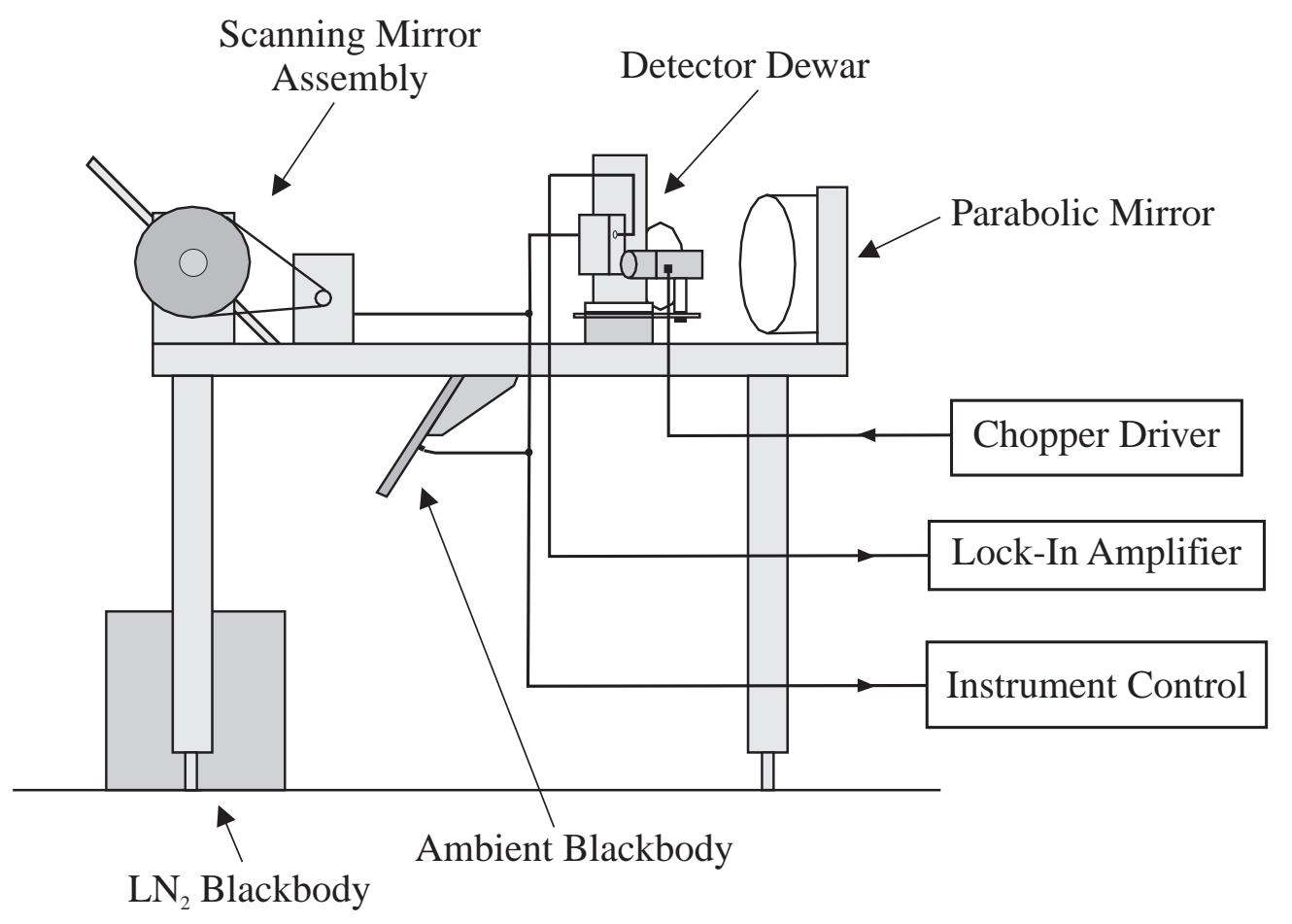

Figure 1. A side view of IRMA 


\section{DATA ANALYSIS}

The primary objective was to compare IRMA II skydips with those obtained with the JCMT SCUBA bolometer camera ${ }^{6}$, which indirectly measures atmospheric water vapor through its contribution to emission at 850 and $450 \mu \mathrm{m}$. Between January and July 2001, 1893 IRMA II skydips were obtained, of which 1026 occurred within twenty minutes of a SCUBA skydip, which takes much longer ( $\sim 7$ minutes). This subset was filtered to remove data of low quality resulting from such things as loss of detector cryogen, obstruction of the beam by a foreign object, and high amounts of atmospheric water vapor. The remaining skydips were then tested for smoothness by non-linear least squares fitting an exponential curve to the data using the CURVEFIT function from the $\mathrm{IDL}^{7}$ library. Departures from smoothness can result from several causes such as thick cloud banks or viewing a nearby cinder cone. This function returns the $\chi^{2}$ of the fit; by inspection it was found that $\chi^{2} \leq 0.001$ produced good fits and this threshold was adopted in the final filtering step. Of the 1026 skydips matched to the SCUBA skydips, 880 , or $\sim 86 \%$, were of high enough quality to be used in the final analysis.

\subsection{Calibration}

Calibration measurements of the blackbodies were obtained at the beginning and end of each skydip. The calibration procedure consisted of observing one blackbody at ambient temperature and one submerged in liquid nitrogen. The calibration measurements, blackbody temperatures, and the IRMA infrared bandpass were then used to establish an instrumental responsivity. This responsivity was subsequently used to calibrate each individual skydip to remove systematic errors, such as those caused by the buildup of dust on the entrance window or the degradation in performance due to loss of vacuum.
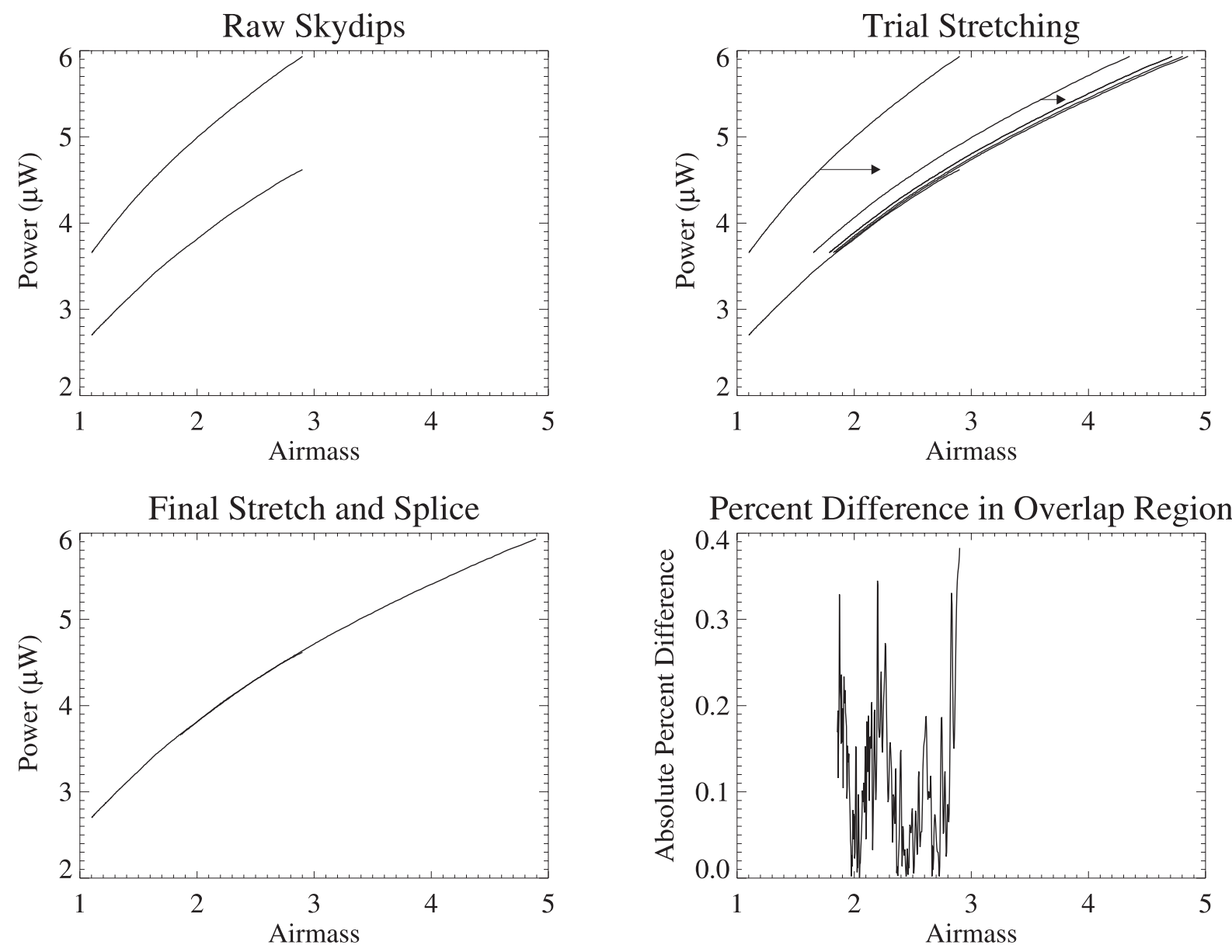

Figure 2. A graphical view of the stretch-and-splice procedure. 


\subsection{Stretch-and-Splice Analysis}

A curve-of-growth represents the spectrally integrated emission from an absorbing molecular species as the total amount of that species is varied in a known way. In the case of the atmosphere, the amount of water vapor is varied by observing the sky at increasing zenith angle, or equivalently, airmass. After calibration, the IRMA skydips can be expressed in terms of incident power as a function of airmass. To simplify the analysis it is assumed that the pressure and temperature profiles, and the scale height of water vapor above Mauna Kea do not vary significantly from day-to-day. Under these assumptions it is possible to construct a composite curve-of-growth from the skydips, by rescaling, or stretching, the horizontal axes of each to reflect the differing amounts of water vapor in the atmosphere at the times of observation, a procedure we term stretch-and-splice.

The stretch-and-splice procedure is shown graphically in figure 2. The upper left panel shows two raw IRMA skydips. In this figure the upper skydip will be stretched to fit with the lower. The upper right panel shows the minimization routine iterating to find the best stretch factor. The lower left panel shows the combined skydips with the upper skydip in the stretched position. The lower portion of the curve-of-growth is produced by taking the lower portion of the lower skydip, averaging the values of the two skydips in the overlapping section, and taking the upper portion of the stretched skydip as it appears. The lower right panel in the figure shows the absolute percent difference between the skydips in the overlap region. The final composite curve-of-growth is generated by repeating this procedure for the remaining 878 skdips, and can then be compared with theoretical models.

Figure 3 shows the resultant composite curve-of-growth (solid line) produced by averaging the overlapping sections of the skydips once they have all been stretched and spliced. A Chebyshev polynomial of degree 6 is fitted to this composite curve to yield a functional form to the data, which is more convenient for comparison with theory. This is shown as the dashed line in the figure (displaced vertically by $0.5 \mu \mathrm{W}$ for clarity). The dotted line shows the difference between the polynomial and the composite curve-of-growth referred to the right hand vertical scale. The low error involved in introducing the Chebyshev polynomial allows its use as a basis curve-of-growth for determining the final stretch factors which are used to calculate the opacity, $\tau_{\text {IRMA }}$, for comparison with other measures of opacity.

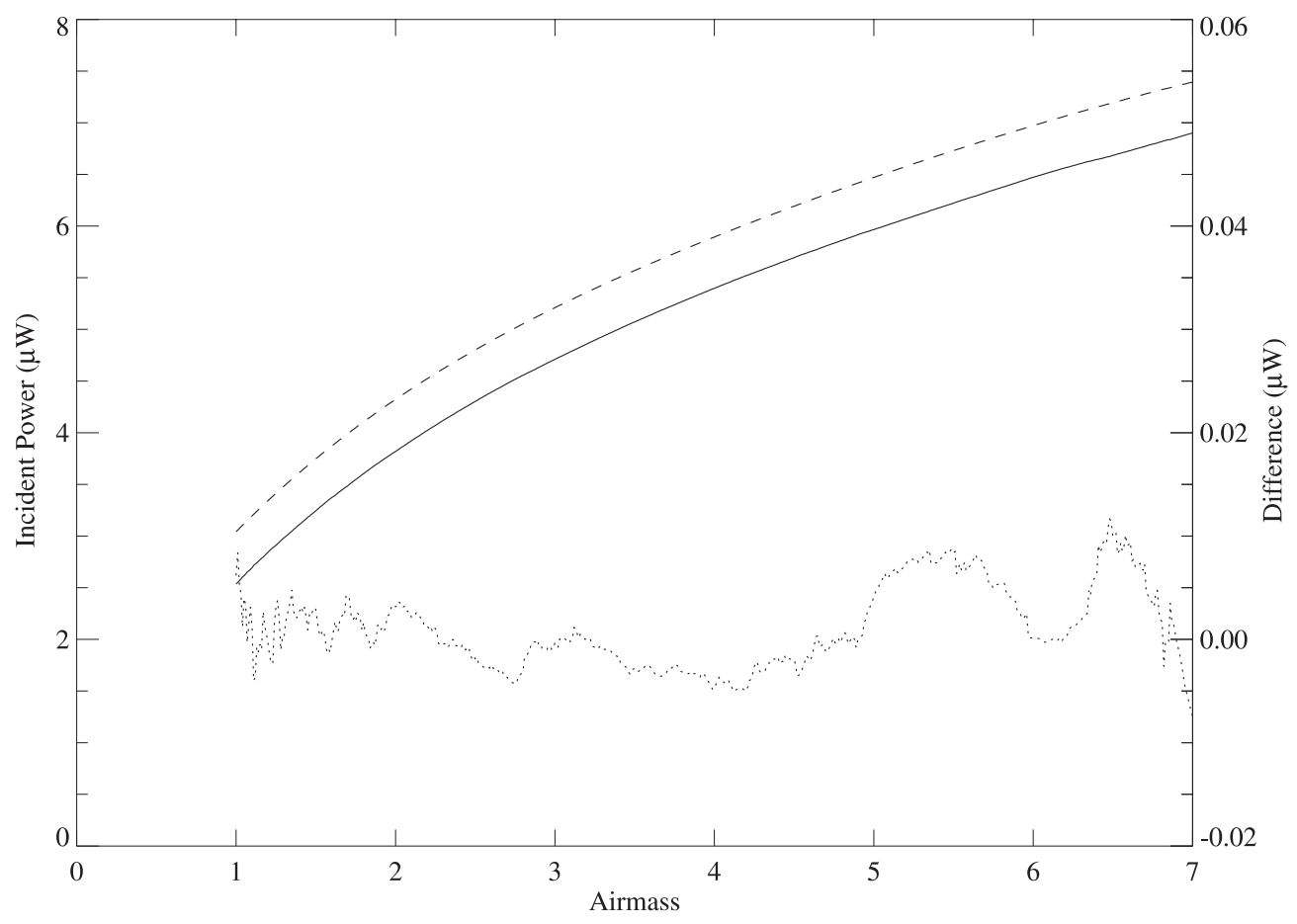

Figure 3. The composite IRMA II curve-of-growth (solid line), the Chebyshev approximation (dashed line; displaced vertically by $0.5 \mu \mathrm{W}$ for clarity) and the difference (dotted line with respect to right hand vertical scale). 


\section{RESULTS}

In the following subsections we compare water vapor column abundances measured by IRMA II with 6 independent measures of water vapor available on the summit of Mauna Kea (e.g. Mauna Kea Weather Center; http://hokukea.soest.hawaii.edu/current) including: the JCMT SCUBA bolometer camera ${ }^{6}$, the California Institute of Technology (CSO) opacity monitors ${ }^{8}$, the JCMT $183 \mathrm{GHz}$ water vapor radiometer ${ }^{9}$ and Hilo-launched radiosonde data. (http://hokukea.soest.hawaii.edu/current/raob/ito/text).

\subsection{IRMA II vs SCUBA $850 \mu \mathrm{m}$}

The standard calibration procedure for SCUBA uses the skydip method to determine the atmospheric transmission in both the 450 and $850 \mu \mathrm{m}$ bands, in order to calibrate the flux from an astronomical source. The SCUBA data are analyzed to produce an opacity, or $\tau_{\text {SCUBA }}$ value ${ }^{6}$. Each IRMA skydip is fitted to the composite curve-of-growth discussed above and its curvature used to determine a corresponding $\tau_{\text {IRMA }}$. If both IRMA and SCUBA are measuring water vapor one would expect the $\tau$ 's to be correlated since both instruments perform skydips in close to the same direction (IRMA is mounted on the carousel floor of the JCMT which generally points in a slightly different azimuth than the telescope, $\sim$ a few degrees).

Figure 4a shows $\tau_{\mathrm{IRMA}}$ plotted against $\tau_{\mathrm{SCUBA}-850 \text {. }}$ Each circle represents the $\tau$ value calculated for a single IRMA skydip. Circles appear in vertical groups because several IRMA skydips can be performed during a single SCUBA skydip. The appearance of columnar grouping in the data indicates that the atmospheric water vapor content is changing appreciably during the few minutes when SCUBA slowly performs a skydip. The linear least squares fit to the data is also shown. Note that most of the circles are tightly clustered about the line, indicating a high degree of correlation between the measurements as expected. The slope of the line shows that the $850 \mu \mathrm{m}$ window saturates slightly faster than the infrared window observed by IRMA. The non-zero intercept of the line indicates that water vapor is not the only species responsible for atmospheric opacity in this window. Atmospheric modeling, currently in progress, shows this is to be expected since the $850 \mu \mathrm{m}$ band contains numerous ozone lines which provide an additional source of opacity not present at $20 \mu \mathrm{m}$.

The $\tau_{\text {SCUBA-850 }}$ values can be converted to water vapor column abundances using an atmospheric model, and the data in figure $4 \mathrm{a}$ can be reformatted, by rescaling the $\mathrm{x}$-axis, to express the power received by the IRMA detector as a function of water vapor (pwv) at the time of observation. Figure $4 \mathrm{~b}$ shows the result of applying a least-squares minimization routine to rescale the composite curve-of-growth (solid curve) to fit the water vapor column abundances derived from the SCUBA $850 \mu \mathrm{m}$ skydips (triangles). The dashed curves represent $\pm 1 \sigma$ deviations from this curve-of-growth, and illustrate the strong correlation between the IRMA and SCUBA $850 \mu \mathrm{m}$ water vapor measurements. The scale factor relating the airmass and pwv scales from this analysis is $\mathrm{F}_{850}=0.49 \pm .04$.
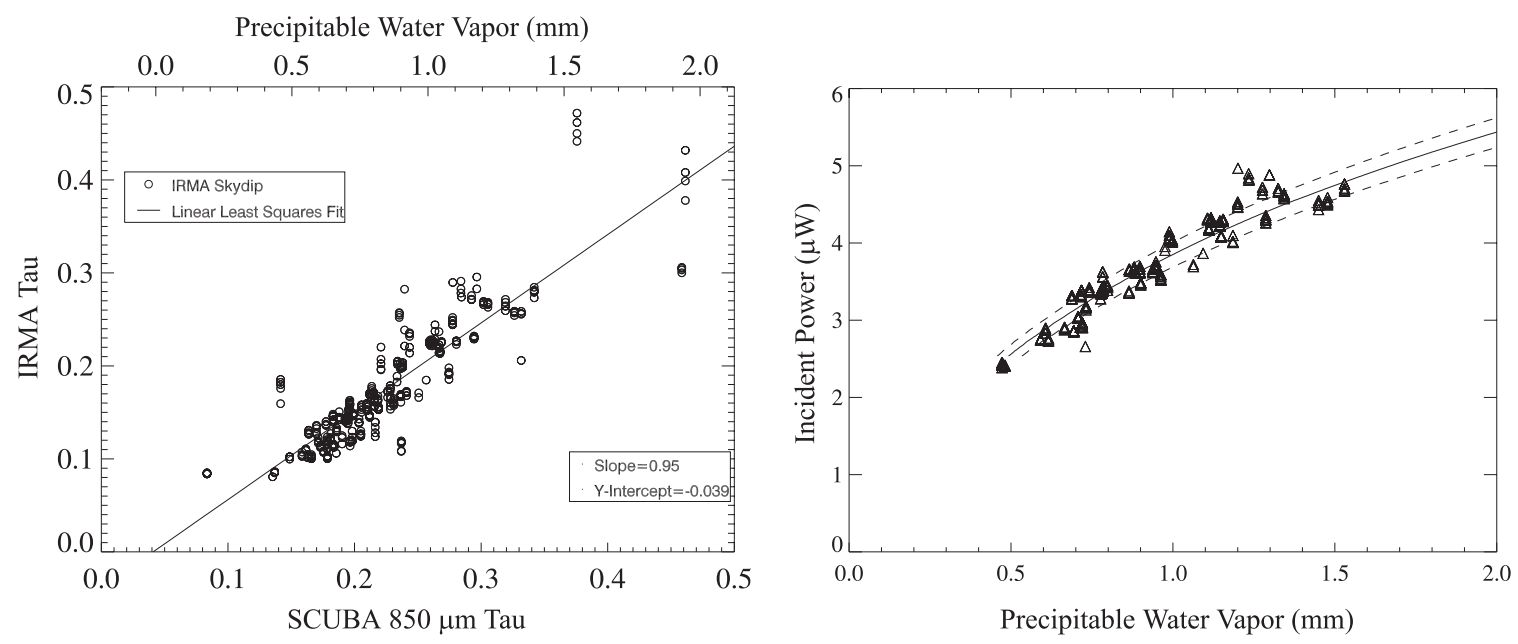

Figure 4a (left) Graph of $\tau_{\text {IRMA }}$ vs $\tau_{\text {SCUBA-850 }}$ and least squares fit. $4 \mathrm{~b}$ (right) IRMA received power calibrated against water vapor column abundance determined from SCUBA $850 \mu \mathrm{m}$ flux (triangles). 


\subsection{IRMA II vs. SCUBA $450 \mu \mathrm{m}$}

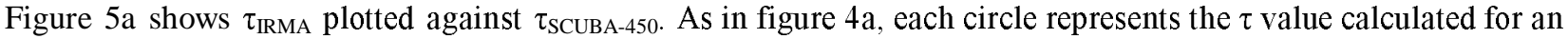
IRMA skydip. Circles again appear in vertical groups because the atmospheric water vapor content changes appreciably during a single SCUBA skydip. The linear least squares fit to the data is shown, and again a high degree of correlation is indicated by the tight clustering of the circles about the line. The slope of the line shows that the $450 \mu \mathrm{m}$ window saturates much faster than the infrared window observed by IRMA. The small, non-zero intercept of the line indicates that water vapor is the main species responsible for atmospheric opacity in this window with a small amount of additional opacity due to numerous ozone lines. However, this additional opacity is less significant since the troposphere has much higher opacity, or lower transmission, in the $450 \mu \mathrm{m}$ band. We are also in the process of modeling the opacity in this spectral region

The $\boldsymbol{\tau}_{\mathrm{SCUBA}-450}$ values can be converted to water vapor column abundance using an atmospheric model, and the data in figure $5 \mathrm{a}$ can be reformatted by rescaling the $\mathrm{x}$-axis to express the power received by the IRMA detector as a function of water vapor (pwv) at the time of observation. Figure 5b shows the result of applying a least-squares minimization routine to rescale the composite curve-of-growth (solid curve) to water vapor column abundances derived from the SCUBA $450 \mu \mathrm{m}$ skydips (triangles). The dashed curves represent $\pm 1 \sigma$ deviations from this curve-of-growth, and illustrate a strong correlation between the IRMA and SCUBA $450 \mu \mathrm{m}$ water vapor measurements. The scale factor relating the airmass and pwv scales from this analysis is $\mathrm{F}_{450}=0.50 \pm .07$.
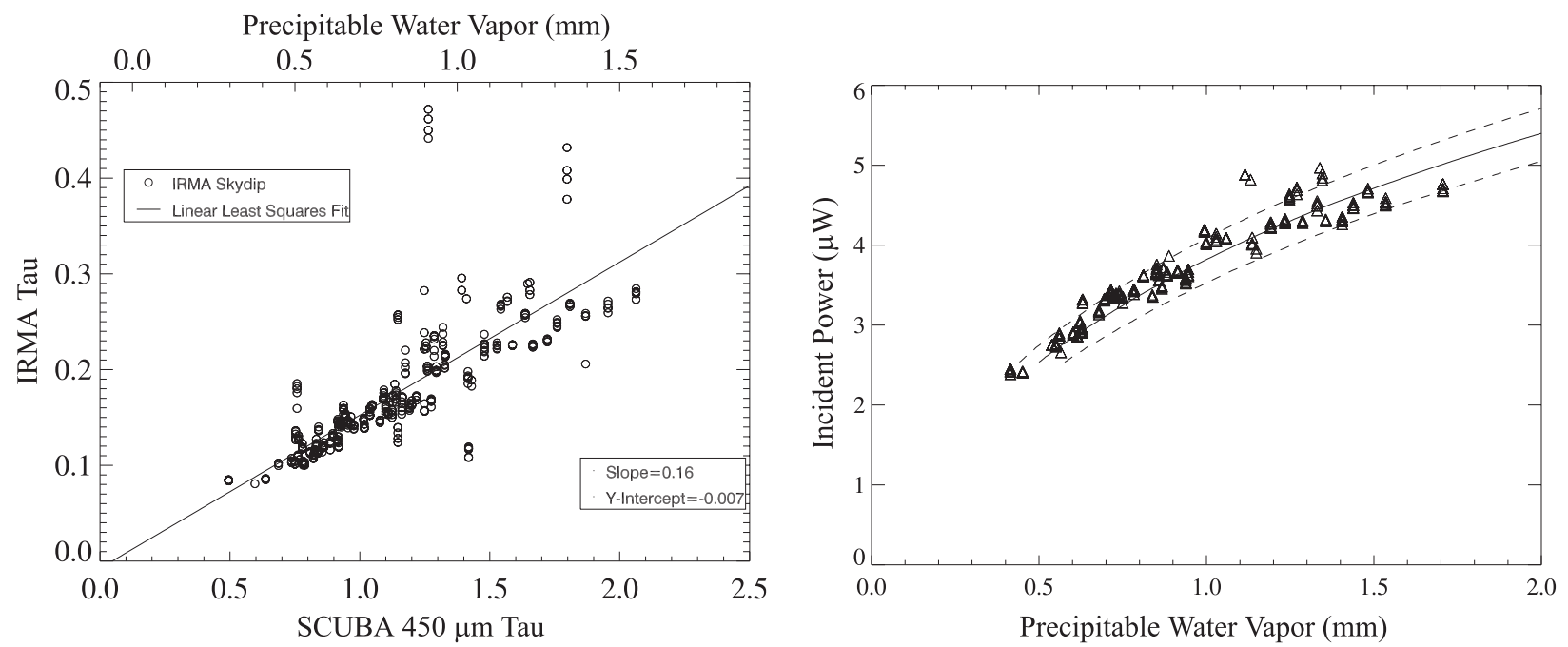

Figure 5a (left) Graph of $\tau_{\text {IRMA }}$ vs $\tau_{\text {SCUBA-450 }}$ and least squares fit. 5b (right) IRMA received power calibrated against water vapor column abundance determined from SCUBA $450 \mu \mathrm{m}$ flux (triangles).

\subsection{IRMA II vs. CSO $225 \mathrm{GHz}$}

The California Submillimeter Observatory (CSO) (http://puuoo.caltech.edu/weather.html) has two radiometers operating at $225 \mathrm{GHz}$ and $350 \mu \mathrm{m}$, which routinely measure the atmospheric opacity in these bands using the skydip method, albeit at a fixed azimuth direction. The CSO data are analyzed in a similar manner to the SCUBA data to produce opacity or $\tau_{\mathrm{CSO}}$ values in the two spectral regions. Conveniently, $\tau_{\mathrm{CSO}-225}$ values are provided along with the $\tau_{\mathrm{SCUBA}}$ values in the SCUBA calibration files. Although in general the CSO radiometers observe a different part of the sky than IRMA, the close proximity of the CSO to the JCMT suggests that $\tau_{\text {IRMA }}$ and $\tau_{\mathrm{CSO}-225}$ might be correlated..

Figure 6a shows $\tau_{\mathrm{IRMA}}$ plotted against $\tau_{\mathrm{CSO}-225}$. As with the SCUBA data, there are several IRMA skydips (represented by circles) for each CSO measurement and again the circles appear in vertical groups because the atmospheric water vapor content changes appreciably during the time of a CSO skydip. The linear least squares fit to the datais also shown. Similar to the previous figures, most of the circles are tightly clustered about the line, indicating a high degree of correlation between the measurements. However, the correlation between $\tau_{\mathrm{IRMA}}$ and $\tau_{\mathrm{CSO}-225}$ is less than with SCUBA, 
which is to be expected since CSO generally observes a different portion of the sky than does IRMA. The slope of the line indicates that CSO operates in a spectral region much less opaque than the infrared region observed by IRMA. The small, non-zero intercept value indicates that water vapor is the main contributor to opacity in the $225 \mathrm{GHz}$ region.

The $\tau_{\mathrm{CSO}-225}$ values can be converted to water vapor column abundance using an atmospheric model. This conversion allows a reformatting of figure $6 \mathrm{a}$ to express the power received by IRMA as a function of water vapor (pwv) at the time of observation as discussed above. Figure $6 \mathrm{~b}$ (similar to figures $4 \mathrm{~b}$ and $5 \mathrm{~b}$ ), shows the result of the least squares minimization routine used to rescale the composite curve-of-growth (solid curve) to water vapor abundances derived from the CSO $225 \mathrm{GHz}$ skydips (triangles). The dashed curves represent $\pm 1 \sigma$ deviations from this curve-of-growth, and illustrate a strong correlation between the IRMA and CSO $225 \mathrm{GHz}$ measurements, but weaker than the correlation with SCUBA as expected. The scale factor relating the airmass and pwv scales from this analysis is $\mathrm{F}_{225}=0.50 \pm .07$.
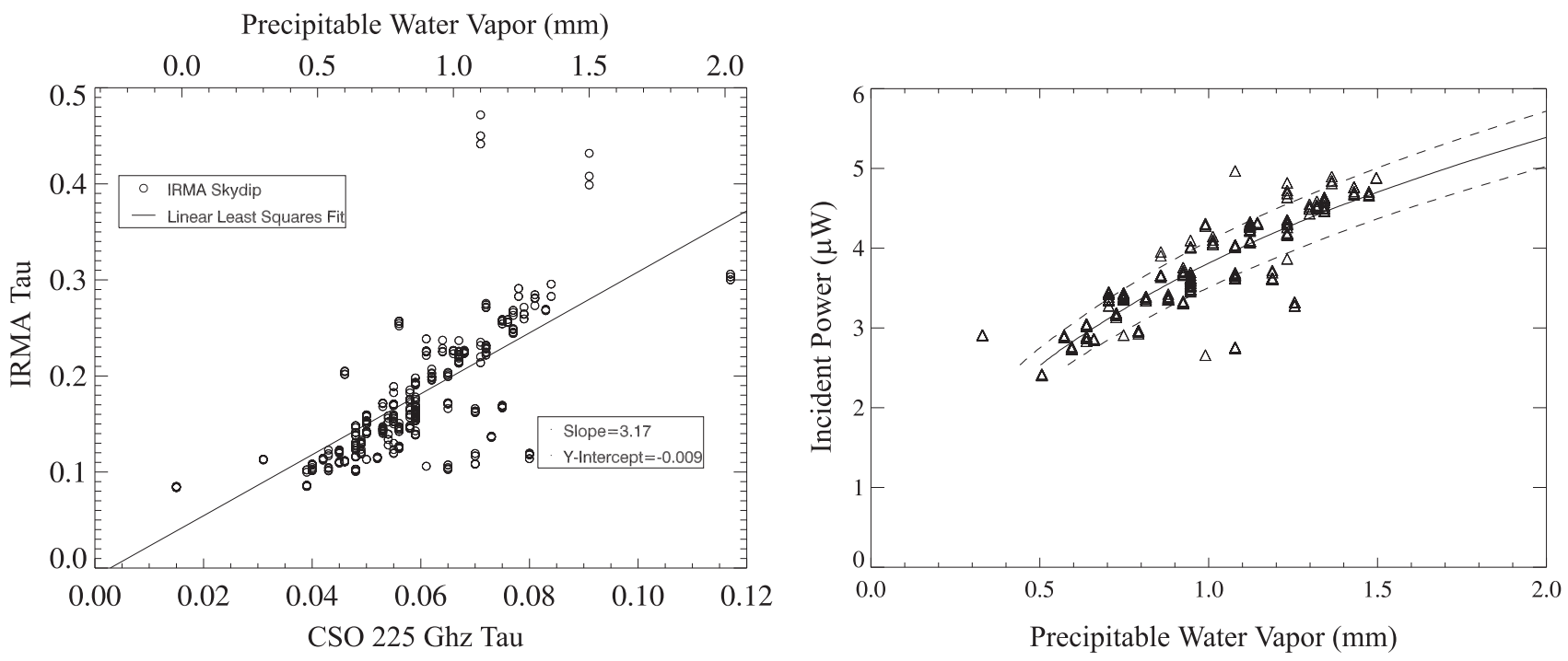

Figure 6a (left) Graph of $\tau_{\text {IRMA }}$ vs $\tau_{\mathrm{CSO}-225}$ and least squares fit. $6 \mathrm{~b}$ (right) IRMA received power calibrated against water vapor column abundance determined from CSO $225 \mathrm{GHz}$ flux (triangles).

\subsection{IRMA II vs. CSO $350 \mu \mathrm{m}$}

Figure 7a shows $\tau_{\text {IRMA }}$ plotted against $\tau_{\mathrm{CSO}-350}$. Again circles, representing individual IRMA skydips, appear in vertical groups because the atmospheric water vapor content changes appreciably during a CSO skydip. The data in this figure show a great deal more scatter than in the previous figures, indicating a low degree of correlation between the measurements of water vapor made by IRMA and those made by CSO $350 \mu \mathrm{m}$. This is primarily due to the high opacity of the $350 \mu \mathrm{m}$ band at these water vapor abundances, as indicated by the $\tau_{\mathrm{CSO}-350}$ values. The linear least squares fit of the data is shown for completeness but its non -zero intercept is not considered physically meaningful.

The $\tau_{\mathrm{CSO}-350}$ values can be converted to water column abundances using an atmospheric model. This conversion allows a reformatting of figure 7a to express the power received by IRMA as a function of water vapor (pwv) at the time of observation as discussed above. Since the scatter in these data resulted in large uncertainties in the least squares minimization routine, figure $7 \mathrm{~b}$ (similar to figure $6 \mathrm{~b}$ ) shows the composite curve-of-growth (solid curve) rescaled by the average of the scale factors determined from the SCUBA 850 and $450 \mu \mathrm{m}$ and CSO $225 \mathrm{GHz}$ data. Even with the large scatter there is reasonable agreement between the water vapor column abundance measurements made by the CSO 350 $\mu \mathrm{m}$ radiometer (triangles) and IRMA. 

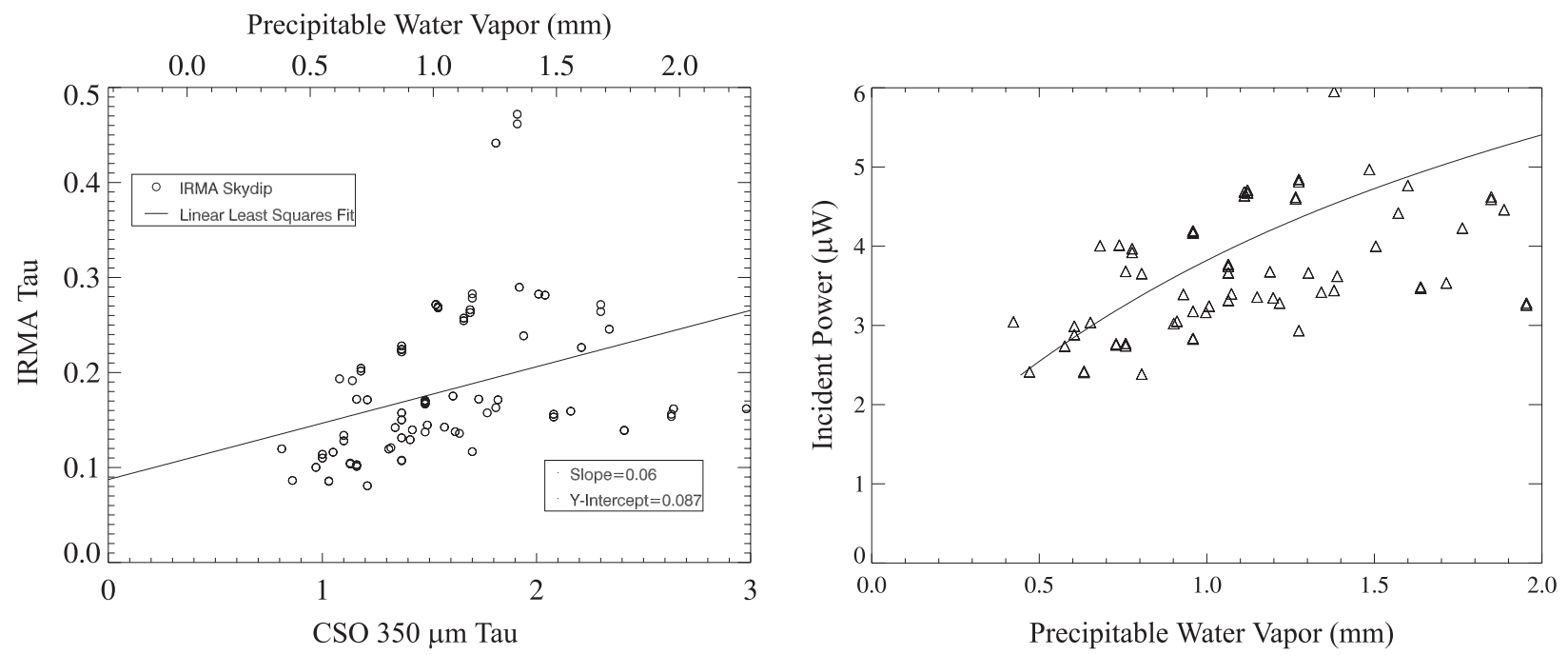

Figure 7a (left) Graph of $\tau_{\text {IRMA }}$ vs $\tau_{\text {CSO-350 }}$ and least squares fit. $7 \mathrm{~b}$ (right) IRMA received power calibrated against water vapor column abundance determined from CSO $350 \mu \mathrm{m}$ flux (triangles).

\subsection{IRMA II vs. $183 \mathrm{GHz}$ Water Vapor Meter}

A water vapor meter (WVM) radiometer has been developed for operation at the $\mathrm{JCMT}^{1}$. This system does not use skydips but rather determines the water vapor abundance along a given line-of-sight from multi-channel radiometric observations of the $183 \mathrm{GHz}$ water line combined with a simple atmospheric model. Although the WVM was not in regular operation between January and July 2001, some archived WVM data which overlaps with IRMA II data is available from the JCMT archive. The WVM data files contain water vapor column abundance and so a figure similar to figure $4 \mathrm{~b}$ can be produced, expressing the power received by the IRMA detector as a function of water vapor amount (pwv) at the time of the observation. Although there is insufficient data to do a least squares analy sis, figure 8 shows the composite curve-of-growth (solid curve) rescaled by the average of the scale factors determined from the SCUBA 850 and $450 \mu \mathrm{m}$ and CSO $225 \mathrm{GHz}$ data. The dashed curves represent $\pm 1 \sigma$ deviations from this curve-of-growth. The relatively low degree of correlation between measurements made by IRMA II and WVM are likely due to the fixed zenith viewing angle of the WVM.

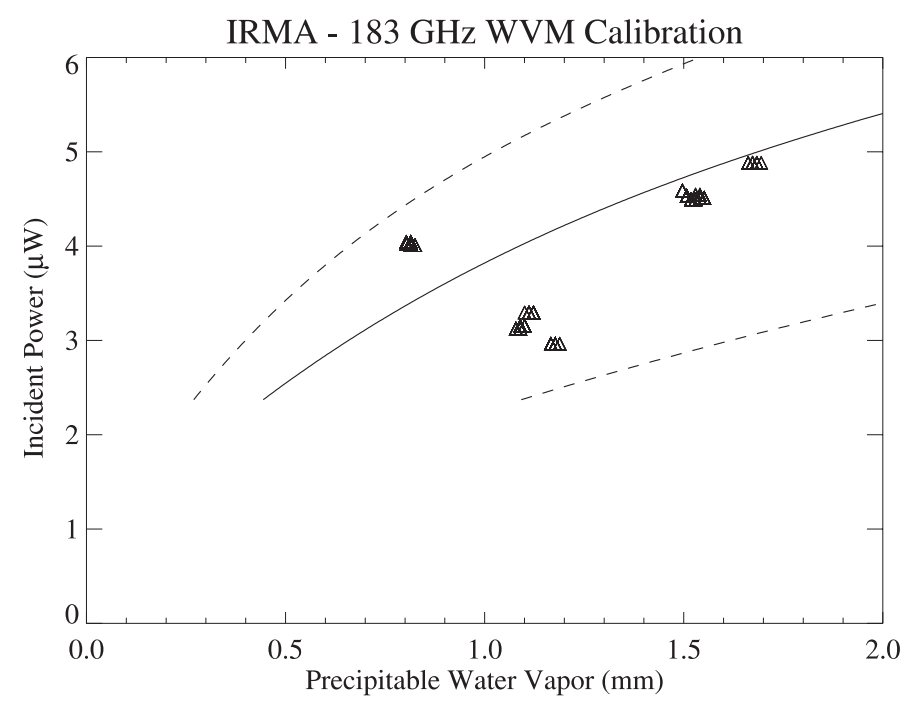

Figure 8. Water vapor column abundance determined from the JCMT $183 \mathrm{GHz}$ water vapor radiometer (triangles) compared to that determined from IRMA calibrated using SCUBA 450 and $850 \mu \mathrm{m}$ and CSO $225 \mathrm{GHz}$ data. 


\subsection{IRMA II vs. Hilo -launched Radiosondes}

Hilo airport launches 2 radiosondes per day (at 0:00 and 12:00 UT), which provide another measure of the water vapor in the atmosphere. The correlation between the IRMA and the radiosonde measurements is likely to be low since the radiosondes are launched into a different part of the atmosphere and tend to carry moisture with them as they rise, which leads to elevated measures of atmospheric water vapor. The radiosonde data can be retrieved from http://hokukea.soest.hawaii.edu/current/raob/ito/text and used to derive water vapor column abundances. IRMA skydips taken within one hour of the radiosonde launches were used in this analysis. Figure 9 is similar to figures $4 \mathrm{~b}-7 \mathrm{~b}$ where the composite curve-of-growth (solid curve) has been rescaled by the average of the scale factors determined from the SCUBA 850 and $450 \mu \mathrm{m}$ and CSO $225 \mathrm{GHz}$ data. The pwv values measured by the radiosonde are shown as tria ngles. Although there is a large spread in these values it is encouraging to see that the scaling factor derived from these independent methods provides a reasonable match to the radiosonde data.

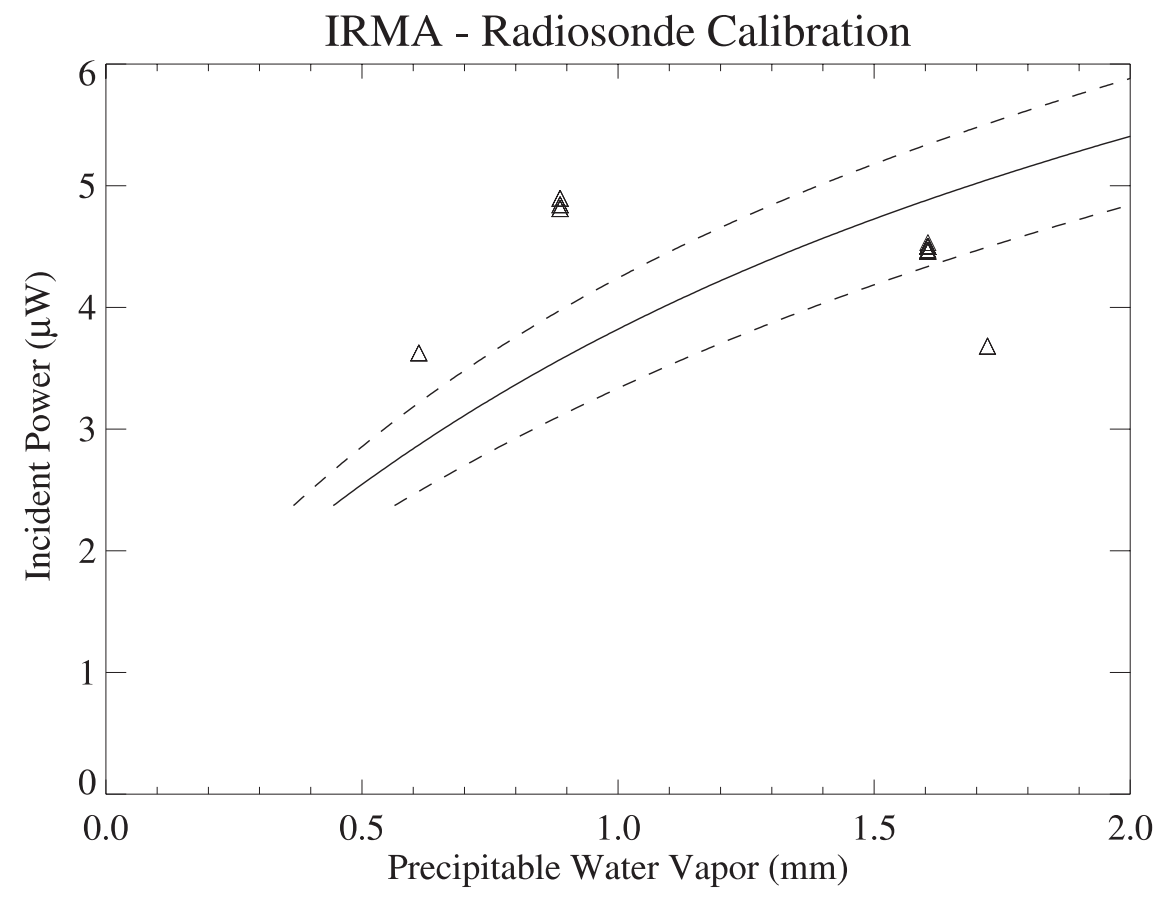

Figure 9 Water vapor column abundance determined from Hilo-launched radiosonde data (triangles) compared to that determined from IRMA calibrated using SCUBA 450 and $850 \mu \mathrm{m}$ and CSO $225 \mathrm{GHz}$ data.

\section{SUMMARY}

Measurements of the column abundance of atmospheric water vapor above Mauna Kea obtained from skydips with an infrared radiometer operating at a wavelength of $20 \mu \mathrm{m}$ show strong correlations with other measures of water vapor available on the summit of Mauna Kea. The results are summarized in Table 1. As expected, the strongest correlations to IRMA measurements are found with the SCUBA 850 and $450 \mu \mathrm{m}$ measures of atmospheric opacity, which are obtained from skydips at approximately the same azimuth angle. The CSO $225 \mathrm{GHz}$ opacity data also show a strong correlation; the increased scatter in these data being attributed to the different azimuth angle of the CSO skydips. The CSO $350 \mu \mathrm{m}$ opacity data show a weaker correlation due to the high opacity of the $350 \mu \mathrm{m}$ band at these water vapor abundances. While the $183 \mathrm{GHz}$ WVM and Hilo-launched radiosonde data are sparse, they are in general agreement with the IRMA results. 


\begin{tabular}{|c|c|c|}
\hline IRMA II Comparison & Scale Factor & Error \\
\hline SCUBA $850 \mu \mathrm{m}$ & 0.49 & $\begin{array}{r}+0.04 \\
-0.04\end{array}$ \\
\hline SCUBA $450 \mu \mathrm{m}$ & 0.50 & $\begin{array}{r}+0.08 \\
-0.06\end{array}$ \\
\hline CSO $225 \mathrm{GHz}$ & 0.50 & $\begin{array}{r}+0.08 \\
-0.06\end{array}$ \\
\hline CSO $350 \mu \mathrm{m}$ & 0.50 & $\begin{array}{r}+2.38 \\
-0.35\end{array}$ \\
\hline \multirow{2}{*}{$183 \mathrm{GHz}$ WVM } & 0.50 & +0.37 \\
& \multirow{2}{*}{0.50} & +0.14 \\
\hline Hilo Radiosondes & 0.50 & -0.10 \\
\hline
\end{tabular}

Table 1. Scaling factors relating IRMA to other measures of water vapor column abundance above Mauna Kea and their associated error.

We are currently developing an atmospheric model for Mauna Kea to provide a theoretical framework for these results. Future work will include incorporating ground pressure and temperature into the data analysis and studying the effects of varying water vapor distributions on the infrared emission.

\section{ACKNOWLEDGEMENTS}

The authors would like to acknowledge the following individuals who have played a significant role in the development of the IRMA project: G.J. Smith for developing the IRMA prototype, I.S. Schofield for developing the software for remote operation, J.R. Ellegood for the design and fabrication of the printed circuit boards, F. Klassen for his machining skills and Ms. E.A. Pope for software support (U of L); Prof. P.A.R. Ade (Cardiff University) for providing the resonant grid infrared filters; Drs. L.W. Avery and P.A. Feldman (HIA, NRC) for their long-standing interest in, and support of, the IRMA project, including arranging for precision machining and provision of key components; Prof. E.I. Robson, Director JAC, and staff of the JCMT for logistical support. DAN acknowledges financial support from NSERC, ASRA and the University of Lethbridge.

\section{REFERENCES}

1. M. Wiedner, "Atmospheric water vapour and astronomical millimeter interferometry", $\mathrm{PhD}$ Thesis, Cambridge, UK, 1998).

2. D. A. Naylor, R. T. Boreiko, T. A. Clark, R. J. Emery, B. Fitton and M. F. Kessler, "Atmospheric emission in the 20-micron window from Mauna Kea", PASP, 96, pp.167-173, 1984.

3. G. J. Smith, "An infrared radiometer for millimeter astronomy", (MSc Thesis, University of Lethbridge, 2001).

4. G. J. Smith, D. A. Naylor and P. A. Feldman, "Measurements of atmospheric water vapor above Mauna Kea using an infrared radiometer", International Journal of Infrared and Millimeter Waves, 22, pp. 661—678, 2001.

5. C. Lee, P.A.R. Ade and C.V. Haynes, "Self supporting filters for compact focal plane designs", Proceedings of the $30^{\text {th }}$ ESLAB Symposium, ESTEC, Noordwijk, The Netherlands, Sept. 1996.

6. W.S. Holland, E.I. Robson, W.K. Gear, C.R. Cunningham, J.F. Lightfoot, T. Jenness, R.J. Ivison, J.A. Stevens, P.A.R. Ade, M.J. Griffin, W.D. Duncan, J.A. Murphy and D.A. Naylor, "SCUBA: a common-user submillimetre camera operating on the James Clerk Maxwell Telescope", Mon. Not. Roy. Astron. Soc., 303, pp. 659-672, 1999.

7. "The Interactive Data Language", Research Systems Inc., 4990 Pearl East Circle, Boulder, CO, 80301, USA.

8. S. J. E. Radford, M. A. Holdaway and J. B. Peterson, "Atmospheric transparency at $350 \mu \mathrm{m}$ wavelength, BAAS, 30, pp. 884, 1998.

9. R. Hills and J. Richer, "ALMA Memo 303: Water vapor radiometers for ALMA", http://www.mma.nrao.edu/memos, 2000. 